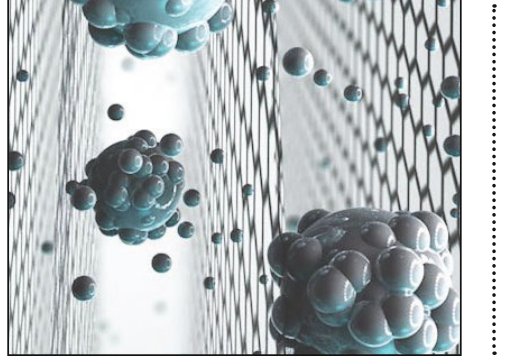

\title{
Will next-generation membranes rise to the water challenge?
}

\author{
By Prachi Patel \\ Feature Editor: Laura Biedermann
}

$\mathrm{W}$ ater is life. Yet for a substance that covers $70 \%$ of the surface of the Earth, it is becoming increasingly precious. The problem is that only $2.5 \%$ of the water on our planet is fresh water. And an exploding world population, growing industrialization, and climate change all threaten this scarce resource.

One in nine people lack access to sufficient, safe drinking water. The world's population could reach 9 billion by 2030, driving up water demand by $30 \%$. Analysts believe current water supplies will meet $60 \%$ or less of global water needs by 2030 . Providing safe water to everyone will require desalinating, purifying, or treating water on a large scale, at low cost, and in a sustainable fashion.

All eyes are on membranes as a possible solution. Membranes can clean, desalt, and treat water. Unlike the large tanks and columns used for traditional water treatments, membranes have a small footprint. "They are scalable and very versatile," said Isabel Escobar, professor of chemical and materials engineering at the University of Kentucky. "They can be packed into fairly small surface areas, and it's fairly easy to increase their size to treat more water."

Membranes have been used to desalinate seawater and treat wastewater on an industrial scale since their invention in the 1960s. Yet, Escobar said, "as excellent as they are, they are still a relatively new technology and have a lot of drawbacks." They are expensive, energy-intensive, and easily soiled. They can suffer from low water flow or lack of selectivity. But by borrowing concepts from biological systems, drawing on novel two-dimensional (2D) materials, and applying advances in chemical synthesis and molecular-level design approaches, researchers hope to crack some of these problems.

Polymer membranes are the current standard technology. They are typically made of a very thin charged polyamide layer deposited on a porous thin-film composite. Depending on their charge and size of their pores, they can remove suspended particles and pathogens (microfiltration); organic matter and smaller pathogens (ultrafiltration); and salts, metals, and ions that cause scaling (nanofiltration and reverse osmosis).

New large-scale desalination plants in Israel, Saudi Arabia, Singapore, and California rely largely on reverse osmosis (RO), in which seawater is forced through semipermeable polymer membranes that block salt and other inorganic impurities. RO requires 3-5 kilowatt hours to produce 1000 liters of drinking water. Much of that energy is needed to push water through the membranes, and it accounts for half the cost of desalination: The US Department of Energy has a goal to decrease the cost of desalination from USD $\$ 2$ to USD $\$ 0.50$ per 1000 liters.
The ideal membrane would let water quickly flow through and hold contaminants back. But there is a tradeoff between a membrane's selectivity and permeability. Membranes that are more porous to water typically also allow other contaminants to pass through. "You can increase a membrane's thickness or density to get higher solute rejection, but then you sacrifice water flux, and the low flux means you need more energy and more membrane area," said Menachem Elimelech, professor of environmental and chemical engineering at Yale University. "We need new ways to make membranes that don't have the that tradeoff."

For desalination, making membranes more selective is key. "Increasing water permeability above levels that are already achievable doesn't affect energy efficiency," said Elimelech. Instead, a critical need is membranes that filter boron and chlorine in seawater. Today, several RO passes are required to reduce those solutes, which slows the process and adds to the cost.

Another problem is that membranes "only remove substances, don't destroy them," said Escobar. "Once their life is over, they are considered hazardous waste." That also leads to a common challenge with membranes: fouling. Impurities clog membrane pores, dragging down performance and lifetime. Industrial plants periodically clean membranes "just like brushing your teeth," said Elimelech. But that takes energy and money, and there is an urgent need for membranes with antifouling surfaces and greater chemical tolerances.

Biology could provide a path forward. Many researchers are placing a bet on aquaporins, the water channels found in cell walls. Discovered in 1993, aquaporins are proteins that form hourglassshaped pores in cell membranes that are just wide enough to let water molecules slip through, but not ions, minerals, proteins, salts, boron, and urea: exactly what a water treatment membrane should do.

Researchers are working on ways to insert aquaporin channels into membranes, for example by loading bacterial aquaporin into vesicles made of lipids or block copolymers - polymers consisting of blocks of distinct monomers. When the vesicles are ruptured on a porous support, the lipid layers or block copolymers arrange themselves, and the porins self-align into channels.

But aquaporin membranes have performed subpar so far. They don't block salt as well as conventional polymer membranes, and the protein degrades after months. Questions also remain as to whether these complex membranes can be made on a large scale at low cost.

Escobar and her colleagues are working on ways to make aquaporin membranes more robust. "The proteins are on the surface and can 
get flattened under water pressure," she said. She and her colleagues are working on ways to anchor them better to the membrane surface so they stay upright and aligned. They are also trying to wrap them in a casing of food-grade gum and other polymers to protect them.

Another idea is to design synthetic water channels that model aquaporin. Researchers have tried this using carbon nanotubes and various peptides by embedding the molecules vertically into a thin film to form pores. But so far it has been difficult to tune the pore sizes to make them narrow enough to block small solutes.

Denmark-based company Aquaporin is already selling the first commercial membrane containing aquaporin channels embedded into polyamide coatings. However, the membranes work as well as traditional RO membranes partly because polyamide is still a large component, said researchers from Dow Water and Process Solutions in a September 2017 review article published in Industrial \& Engineering Chemistry Research (doi:10. acs.iecr.7b02411). "It will be interesting to see if customers are willing to pay a premium for this technology, and whether it can be priced competitively and produced cost-effectively for widespread market adoption."

Low-cost nanomaterials have also taken the membrane world by storm. In April, a research team from Hong Kong and Saudi Arabia reported a novel nanoporous membrane made of a 10 - $\mu \mathrm{m}$-thick carbon nanofiber layer deposited on a porous yttrium-stabilized zirconia ceramic substrate. The membrane blocked $99.9 \%$ of salt and had 3-20 times higher freshwater flux than conventional polymer membranes.

Atoms-thick materials such as graphene and molybdenum disulfide are exciting because their flat sheets can be easily stacked with proper spacing to make thin films, said Baoxia Mi, a civil and environmental engineering professor at the University of California, Berkeley. "The layers create unique 2D nanochannels that give a transport path for water molecules," she said. Plus, it is easy to tailor their properties: attach functional groups to their surface to make them more hydrophilic, or attach catalysts that spur reactions to break down contaminants.

Graphene oxide is inexpensive to produce at large scale. It is chemically stable, has antimicrobial properties, and its smooth hydrophilic surface is inherently resistant to fouling. But a major issue is that thin graphene oxide films swell in water, widening the nanochannels and allowing ions to flow through with water. Andre Geim and his colleagues at The University of Manchester have shown that locking the graphene layers in epoxy could physically restrict swelling, as can blending graphene flakes with the graphene oxide sheets.

Yet, researchers are far from proving that 2D nanomaterial membrane technology works at large scale and is eco- nomically viable. With graphene oxide membranes, for instance, "it's very difficult to get even a $1-\mathrm{cm}$ by $1-\mathrm{cm}$ membrane that's defect-free," said Elimelech. "And to take it farther for large areas of thousands of square meters needed for desalination plants would be a major challenge."

Graphene-polymer composite membranes might have an advantage, believes Lauren Greenlee, a chemical engineer at the University of Arkansas. Her group makes membranes by dispersing graphene oxide particles in polymer matrices, a versatile method. "You can modify membrane properties by changing graphene oxide flake size or the acid you use in the casting solution or by surface functionalization," she said.

These membranes are promising for removing organic contaminants. In December 2017, Greenlee's group reported a membrane made of graphene oxide and chitosan, a biopolymer derived from crustacean shells. The membrane blocked $95 \%$ of blue dye molecules, and its performance was linked to its electrostatic properties, not just size exclusion of contaminants. "We're seeing some really interesting things," said Greenlee. "Some nice properties of graphene oxide, like antifouling, seem to be transferred to the membranes."

Mi and others are exploring 2D molybdenum disulfide, which does not swell in water but is more expensive and not as stable as graphene oxide. Escobar, meanwhile, is studying phosphorene, atom-thick phosphorus sheets that are more electrically conductive than graphene. "We are trying to determine if it has potential to be photocatalytically active," she said, which could lead to a membrane that degrades impurities and resists fouling.

Any new membrane technology will have to deal with fouling. "Once we create next-generation membranes, we need to think about surface modification to minimize fouling," said Elimelech. Making membranes hydrophilic reduces fouling because proteins and organic compounds are hydrophobic. The most promising approach is to cover a membrane's surface with a brush-like layer of hydrophilic polyethylene or zwitterionic polymers. This is commonly done to prevent bacterial growth and protein buildup in biomedical devices. "But membrane companies do a simple proprietary coating and just learn to live with fouling," he said. "They have perfected their membrane manufacturing protocol and made membranes cheap. It's a competitive market, and changing the manufacturing process is difficult."

In the end, this manufacturing inertia could be a big hurdle. But overcoming it might be critical. The lack of clean water is one of the biggest challenges we face today. Membrane technologies that work on large scale at low cost using little energy will be a must to quench the world's thirst. $\square$ 